\title{
Pulmonary contusion during the COVID-19 pandemic: challenges in diagnosis and treatment
}

\author{
Yongyong Wang ${ }^{1} \cdot$ Chenxi Zeng $^{1} \cdot$ Liming Dong $^{2} \cdot$ Changyu Liu $^{1} \cdot$ Yixing Cai ${ }^{1} \cdot \mathrm{Ni}_{\text {Zhang }}{ }^{1} \cdot$ Xiangning Fu $^{1}$
}

Received: 20 May 2020 / Accepted: 22 June 2020 / Published online: 22 July 2020

(c) Springer Nature Singapore Pte Ltd. 2020

\begin{abstract}
Managing patients with pulmonary contusion safely and effectively during the coronavirus disease 2019 (COVID-19) pandemic is challenging. This retrospective study analyzes the clinical data of 29 consecutive patients with pulmonary contusion, including two with COVID-19, at Tongji Hospital, Wuhan, China, in January and February, 2020. We analyzed the clinical manifestations, laboratory test results, computed tomography (CT) images, treatment, and clinical outcomes. The two patients with pulmonary contusion and COVID-19 had increased leukocyte and neutrophil counts, similar to the patients with pulmonary contusion alone. Interestingly, both these patients had subpleural ground glass opacity on CT images as a typical manifestation of COVID-19. All 29 patients were treated conservatively, including with closed thoracic drainage, instead of with thoracotomy. Six patients died of ARDS or craniocerebral injury, but the others stabilized. During the COVID-19 pandemic, patients with pulmonary contusion should be tested for SARS-CoV-2 and unless critical, thoracotomy should be avoided.
\end{abstract}

Keywords Pulmonary contusion · COVID-19 · Treatment

SARS-CoV-2 is highly contagious and the COVID-19 pandemic endangers overall health, posing new challenges for managing other diseases and events, including chest trauma [1]. Chest trauma combined with pulmonary contusion is one of the most common thoracic surgical emergencies [2]. The laboratory testing results and computed tomography (CT) images of patients with pulmonary contusion show some similarities to those of patients with acute pneumonia [3]. A recent case report demonstrated that the CT findings of some lung contusions are similar to those of COVID-19 [3]. Thus, the differential diagnosis of lung contusion and

Yongyong Wang and Chenxi Zeng contributed equally to this study.

Xiangning $\mathrm{Fu}$

fuxiangning06@163.com

1 Department of Thoracic Surgery, Tongji Hospital, Tongji Medical College, Huazhong University of Science and Technology, 1095\# Jiefang Avenue, Wuhan City, Hubei Province, China

2 Department of Trauma Surgery, Tongji Hospital, Tongji Medical College, Huazhong University of Science and Technology, 1095\# Jiefang Avenue, Wuhan City, Hubei Province, China
COVID-19 is required, highlighting the challenges of treating pulmonary contusion amid the COVID-19 outbreak. We analyzed, retrospectively, the clinical and laboratory characteristics, treatment, and outcomes of a consecutive cohort of 29 patients with pulmonary contusion, including two also confirmed to have COVID-19, who were admitted to the Tongji Hospital, Wuhan, China, between January, 23 and February 28, 2020.

Table 1 summarizes the clinical manifestations of the 29 patients. Twenty-seven patients had only pulmonary contusion, with a clear history of trauma, including car accident injuries $(n=16)$, fall-related injuries $(n=7)$, and blunt force injury $(n=4)$. Two patients who had pulmonary contusion combined with COVID-19, had fall-related injuries and a history of COVID-19 exposure. Fever developed 38-59 h post-injury in 6 of the 27 patients with pulmonary contusion; whereas, the 2 patients with COVID-19 had a fever before their injury. Twenty-four of the 27 patients with pulmonary contusion had increased leukocyte and neutrophil counts (88.9\%), and 26 had a low lymphocyte count (96.3\%). The two patients with COVID-19 had increased leukocyte and neutrophil counts, and one had a low lymphocyte count.

The CT images of the 27 patients with only pulmonary contusion showed increased lung markings, scattered 
Table 1 Clinical course of the 29 patients with pulmonary contusion including 2 with COVID-19

\begin{tabular}{|c|c|c|}
\hline Variables, $n(\%)$ & $\begin{array}{l}\text { Pulmonary contusion } \\
(n=27)\end{array}$ & $\begin{array}{l}\text { Combined with } \\
\text { COVID-19 } \\
(n=2)\end{array}$ \\
\hline Age & $40(23-72)$ & $46-59$ \\
\hline \multicolumn{3}{|l|}{ Gender } \\
\hline Male & $19(70.4)$ & $1(50)$ \\
\hline Female & $8(29.6)$ & $1(50)$ \\
\hline \multicolumn{3}{|l|}{ Symptoms } \\
\hline Fever & $6(22.2)$ & $2(100)$ \\
\hline Cough & $8(29.6)$ & $2(100)$ \\
\hline \multicolumn{3}{|l|}{ Contact history } \\
\hline Yes & $4(14.8)$ & $2(100)$ \\
\hline No & $23(85.2)$ & $0(0)$ \\
\hline \multicolumn{3}{|l|}{ Leukocytes } \\
\hline Increase & $24(88.9)$ & $2(100)$ \\
\hline Decrease & $0(0)$ & $0(0)$ \\
\hline Normal & $3(11.1)$ & $0(0)$ \\
\hline \multicolumn{3}{|l|}{ Neutrophils } \\
\hline Increase & 24 (88.9) & $2(100)$ \\
\hline Decrease & $0(0)$ & $0(0)$ \\
\hline Normal & $3(11.1)$ & $0(0)$ \\
\hline \multicolumn{3}{|l|}{ Lymphocytes } \\
\hline Increase & $0(0)$ & $0(0)$ \\
\hline Decrease & $26(96.3)$ & $2(100)$ \\
\hline Normal & $1(3.7)$ & $0(0)$ \\
\hline
\end{tabular}

Fever was defined as a body temperature $>37.3{ }^{\circ} \mathrm{C}$

The normal ranges of leukocyte, neutrophil and lymphocyte counts were $3.5-9.5 * 10^{9} / \mathrm{L}, \quad 40.0-75.0 * 10^{9} / \mathrm{L}$ and $1.1-3.2 * 10^{9} / \mathrm{L}$ respectively. Cell numbers lower than the normal minimum were regarded as a decrease. Cell numbers higher than the normal maximum were regarded as an increase

plaque-like shadows, and subsegmental areas of consolidation. Four (14.8\%) of these patients had pneumothorax, $3(11.1 \%)$ had hydrothorax, $4(14.8 \%)$ had subcutaneous emphysema, and 5 (18.5\%) had rib fracture. Two patients with both pulmonary contusion and COVID-19 had plaque-like shadows in the lower lobes with ground glass opacity (GGO) beside the lesion area, being a feature of COVID-19 infection (Fig. 1). None of the 27 patients without COVID-19 had GGO on their CT images.

All 27 patients with pulmonary contusion alone were treated symptomatically. We took more conservative measures than usual, performing closed thoracic drainage only for patients who had pneumothorax or hydrothorax. None of the patients underwent thoracotomy, although ten required emergency surgery, for craniocerebral injury $(n=4)$, fracture requiring open reduction $(n=3)$, rupture of abdominal organs $(n=2)$, and spinal injury $(n=1)$. Two patients died of acute respiratory distress syndrome (ARDs) and three died of craniocerebral injury. Twenty patients were discharged from hospital and two remained as in-patients in a stable condition at the time of manuscript submission.

One of the two pulmonary contusion patients with COVID-19 died of severe brain injury the day after admission. The other patient, a 49-year-old man, was admitted to hospital in shock with a Glasgow coma score of only 3. Brain injury, pulmonary contusion, multiple rib fractures, pneumothorax, and paraplegia were diagnosed and the emergency physicians performed endotracheal intubation and closed drainage of the right thoracic cavity. He was transferred to the ward on mechanical ventilation and received analgesia, anti-shock, and other supportive treatment. On day 1 , chest $\mathrm{CT}$ revealed pulmonary contusion in both lower lobes and multiple subpleural GGOs (Fig. 2a). He had a fever of more than $39^{\circ} \mathrm{C}$. On day 3 , his Glasgow coma score was 7 , but he still relied on the ventilator. His chest CT scan showed a clear sign of COVID-19 infection (Fig. 2b). He was given the antiviral agents, oseltamivir and lopinavir/ritonavir, as well as corticosteroid impulse therapy, antibacterial agents, low-molecular-weight heparin, and the Chinese medicine Xuebijing as injections. After a series of treatments, his body temperature returned to normal on day 9. Repeat chest CT showed remarkable improvement of the COVID-19 infection signs (Fig. 2c). The COVID-19 RTPCR test was negative twice, on days 14 and 17. At the time of writing, he was in a stable condition and had been transferred to the rehabilitation department.
Fig. 1 Chest computed tomography $(\mathrm{CT})$ image showing ground glass opacity in the right lung beside the pulmonary contusion areas in a 56-year-old patient brought to hospital $1 \mathrm{~h}$ after a high fall injury. COVID19 was subsequently diagnosed. The patient succumbed to the severe brain injury the next day
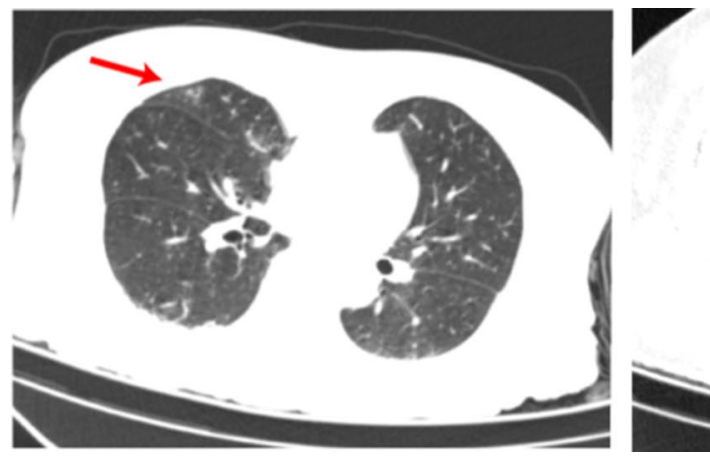
A
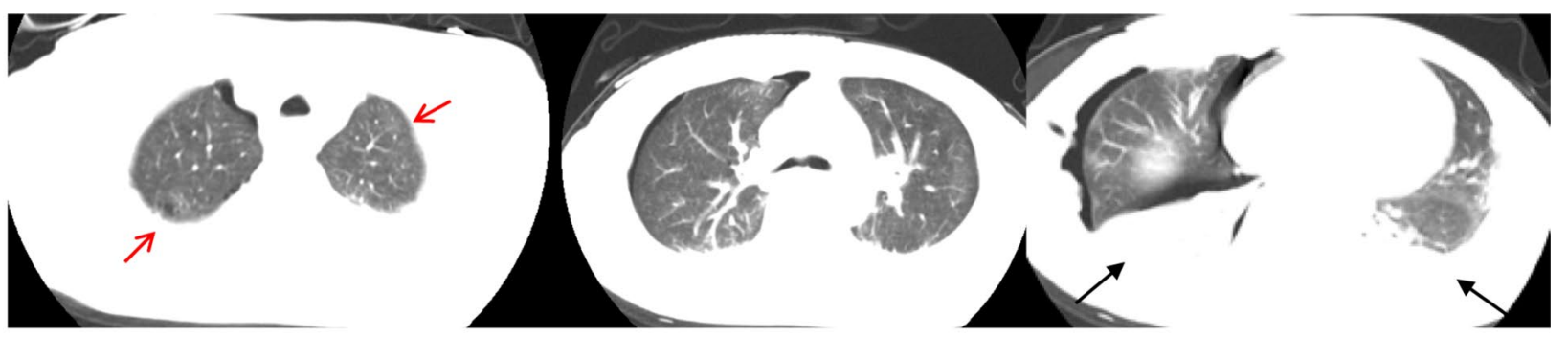

B

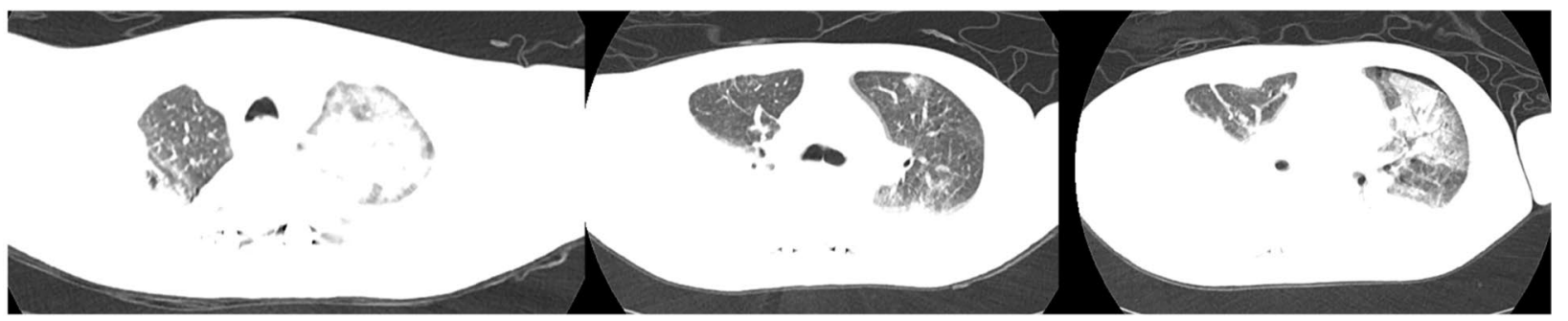

C

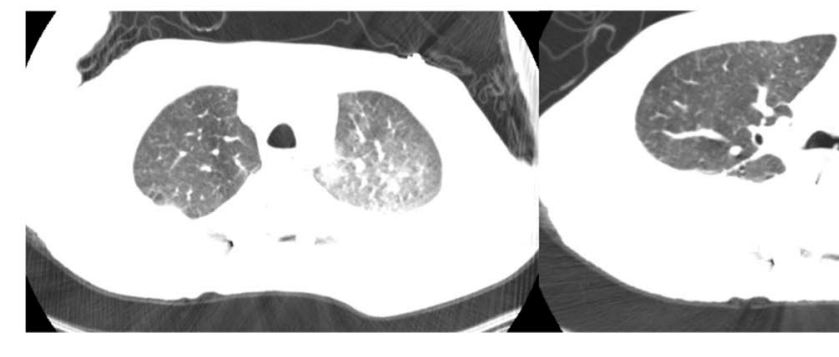

Fig. 2 On day 1 after a high fall injury, chest computed tomography (CT) image showed plaque-like shadows in both lower lobes (black arrow), and ground glass opacity in both upper lobes (red arrow) in a 49-year-old man (a). On day 3, CT showed consolidation of the upper

Pulmonary contusion is caused by an acute chest injury; most often motor vehicle accidents and falls from heights. The major clinical manifestations are chest pain, dyspnea, and hemoptysis [2, 4]. Patients with COVID-19 generally have a clear history of close contact with a confirmed case. The early clinical manifestations are fever and dry cough, sometimes accompanied by muscle aches, fatigue, and diarrhea [5]. Fever is often the first symptom [6]. However, fever develops in some patients with pulmonary contusion, $48-72 \mathrm{~h}$ post-injury. Six of the 27 patients with pulmonary contusion without COVID-19 had fever, which developed 38-59 h post-injury. The two pulmonary contusion patients exposed to COVID-19 had fever before the injury. Establishing the contact history, understanding the characteristics and symptoms, and identifying the sequence of symptoms and injuries are important markers for the differential diagnosis.

Several studies have found decreased or normal leukocyte counts and decreased lymphocyte counts in patients with COVID-19 [4, 7]. It is noteworthy that our two patients with pulmonary contusion combined with COVID-19 had increased leukocyte and neutrophil lobes and diffused pulmonary infection, indicating rapid progression of the COVID-19 infection (b). On day 12, CT showed almost complete resolution of the consolidation in the upper lobes and a reduction in the lesion area in both lower lobes (c)

counts, which is uncommon in COVID-19. Hence, physicians should be vigilant not to overlook the possibility of COVID-19 in a patient with pulmonary contusion because of increased leukocyte and neutrophil counts.

Chest CT is a highly sensitive examination for lung lesions. Typical CT images of pulmonary contusion are increased lung markings, accompanied by a diffuse plaquelike or flocculent shadow [8]. CT images of patients with COVID-19 show the coexistence of various lesions in the lung, such as GGO, consolidation, and nodules $[9,10]$. CT images of patients with mild pulmonary contusion may be similar to those of patients with COVID-19. In this study, the CT images of the two pulmonary contusion patients with COVID-19 showed subpleural GGO out of the pulmonary contusion areas. We previously reported seven cases of confirmed COVID-19 infection in the perioperative period after lung resection, in which emerging multifocal GGOs were seen on the CT images. Therefore, CT images of patients with pulmonary contusion should be interpreted carefully and GGOs must not be overlooked during the pandemic. 
We treated our surviving pulmonary contusion patient who also had COVID-19 with corticosteroids. However, the efficacy of corticosteroids for influenza-associated pneumonia or ARDS, and especially for COVID-9 infection is controversial. The Infectious Diseases Society of America (IDSA) recommended against treating influenza with corticosteroid therapy, unless it was clinically indicated for other reasons [11]. However, several studies have found that the early administration of corticosteroids can improve the prognosis of patients with pulmonary contusion [10, 12], and on this basis, we administered corticosteroids to control the symptoms. To avoid secondary lung injury, we adjusted the ventilator settings to low tide air volume $(6 \mathrm{~mL} / \mathrm{kg}$ of predicted body weight) and positive end-expiratory pressure (PEEP), which has been proven appropriate for ARDs caused by virus infection [13]. We also gave the patients a Chinese medicine called Xuebijing. Chen et al. [14] reported that Xuebijing injections can improve the prognosis of patients with severe pulmonary contusion by regulating the immune system. Xuebijing injection is also used widely in the treatment of COVID-19 in China.

In our previous study, the incidences of severe and critical disease among thoracic surgery patients were 27.3 and $36.4 \%$, respectively [15], suggesting that thoracic surgery can compromise outcomes. We recommended that thoracotomy be performed with extreme caution in pulmonary contusion patients with COVID-19 infection. Furthermore, to reduce postoperative risk and avoid unnecessary exposure to the novel coronavirus, pulmonary contusion patients should be treated conservatively if possible.

The limitations of this study include its small sample size, especially the fact that there were only two patients with pulmonary contusion combined with COVID-19, indicating bias and limited generalizability in the study results. Further follow-up is also still needed.

In conclusion, patients with pulmonary contusion and those with COVID-19 may present some similarities during their clinical course. Treatment for patients with pulmonary contusion combined with COVID-19 infection should be considered comprehensively. During the pandemic, it is essential to balance the benefit of surgery with the risks of unnecessary exposure to the virus for healthcare professionals and patients by changing the management principles. Thoracotomy should be avoided and the possibility of COVID-19 infection coexisting with pulmonary contusion must be borne in mind.

\section{Compliance with ethical standards}

Conflict of interest We have no conflicts of interest to declare in association with the present study.

\section{References}

1. WHO. Coronavirus disease (COVID-2019) situation reports. Available at: http:/www.who.int/emergencies/disease/novel-coron avirus-2019/situation-reports. Accessed 10 Mar 2020.

2. Rendeki S, Molnar TF. Pulmonary contusion. J Thorac Dis. 2019;11(Suppl 2):S141-51.

3. Chen LR, Chen ZX, Liu YC, Peng L, Zhang Y, Xu Q, et al. Pulmonary contusion mimicking COVID-19: a case report. World J Clin Cases. 2020;8(8):1554-60.

4. Wanek S, Mayberry JC. Blunt thoracic trauma: flail chest, pulmonary contusion, and blast injury. Crit Care Clin. 2004;20(1):71-81.

5. Huang CL, Wang YM, Li XW, Ren LL, Zhao JP, Hu Y, et al. Clinical features of patients infected with 2019 novel coronavirus in Wuhan, China. Lancet. 2020;395(10223):497-506.

6. Cheng ZJ, Shan J. 2019 Novel coronavirus: where we are and what we know. Infection. 2020.

7. Guan WJ, Ni ZY, Liang WH, Ou CQ, He JX, Liu L, et al. Clinical characteristics of coronavirus disease 2019 in China. N Engl J Med. 2020.

8. Kaewlai R, Avery L, Asrani A, Novelline R. Multidetector CT of blunt thoracic trauma. Radiographics. 2008;28(6):1555-70.

9. Cai Y, Hao Z, Gao Y, Ping W, Wang Q, Peng S, et al. COVID-19 in the perioperative period of lung resection: a brief report from a single thoracic surgery department in Wuhan, China. J Thorac Oncol, 2020. pii: S1556-0864(20)30298-7.

10. Svennevig JL, Pillgram-Larsen J, Fjeld NB, Birkeland S, Semb G. Early use of corticosteroids in severe closed chest injuries: a 10-year experience [J]. Injury. 1987;18:309-12.

11. Uyeki TM, Bernstein HH, Bradley JS, Englund JA, File TM, Fry $\mathrm{AM}$, et al. Clinical practice guidelines by the Infectious Diseases Society of America: 2018 update on diagnosis, treatment, chemoprophylaxis, and institutional outbreak management of seasonal influenza. Clin Infect Dis. 2019;68(6):895-902.

12. Akdemir HU, Güzel A, Katı C, Duran L, Alaçam H, Gacar A, et al. The evaluation of different treatment protocols for traumainduced lung injury in rats[J]. J Thorac Dis. 2014;6:66-73.

13. Briel M, Meade M, Mercat A, Brower RG, Talmor D, Walter $\mathrm{SD}$, et al. Higher vs lower positive end-expiratory pressure in patients with acute lung injury and acute respiratory distress syndrome: systematic review and meta-analysis. JAMA. 2010;303(9):865-73.

14. Chen Y, Tong H, Zhang W, et al. Curative effect of Xuebijing injection on severe pulmonary contusion. J Tradit Chin Med. 2013;33:743-51.

15. Peng S, Huang L, Zhao B, Zhou S, Braithwaite I, Zhang N, et al. Clinical course of coronavirus disease 2019 in 11 patients after thoracic surgery and challenges in diagnosis. J Thorac Cardiovasc Surg. 2020 (S0022-5223(20)30859-X).

Publisher's Note Springer Nature remains neutral with regard to jurisdictional claims in published maps and institutional affiliations. 